\title{
Mesoporous titania nanoparticles as photocatalyst for degradation of 2-chlorophenol
}

\author{
N. F. Jaafar ${ }^{*}$ and N. A. Marfur \\ School of Chemical Sciences, Universiti Sains Malaysia, 11800 USM Penang, Malaysia.
}

ABSTRACT - Mesoporous titania nanoparticles (MTN) was successfully prepared by microwaveassisted menthod. The performance of MTN was compared with degussa P25 (commercial $\mathrm{TiO}_{2}$ ) on photocatalytic degradation of 2-chlorophenol (2-CP). Both catalysts were characterized by XRD, FTIR, UV-Vis DRS and surface area analysis. The characterization data indicated that MTN has higher surface area and lower particle size than P25. The 2-CP was successfully degraded completely under UV light irradiation despite of having a slightly higher band-gap value compared with P25. This study demonstrated that MTN shows a good potential as a photocatalyst.

\author{
ARTICLE HISTORY \\ Received: 1 March 2021 \\ Revised: 26 April 2021 \\ Accepted: 29 April 2021 \\ KEYWORDS \\ Mesoporous Titania \\ Photocatalytic degradation \\ 2-chlorophenol
}

\section{INTRODUCTION}

The role of water as one of the importance sources in living life is irrefutable because there is only $3 \%$ of fresh water is available and less than $1 \%$ of all fresh water is readily accessible for human use. However, less than $0.007 \%$ of all the water on earth is available to drink and 1 in 5 do not have access to safe water drinking. According to the UN, every 15 seconds a child dies from a water-related disease. Nowadays, this situation getting more critical since the rapid growth in the industry becoming the biggest contributor to environmental pollution. Among them, there are massive type of organic pollutants such as biphenyls, organic dyes and phenols [1]. 2-Chlorophenol (2-CP) is one of the halogenated phenolic compound which widely used in the industries for production of many kinds of compounds such as herbicides, pesticides, cosmetics and pharmaceutical products. However, it is classified as an extremely dangerous compound due to its high toxicity, low biodegradability, tendency to bioaccumulate in various water bodies and persistence in the environment [2]. It also very harmful towards humans since they may cause histopathological alterations, genotoxicity, skin irritation and carcinogenicity [3]. In the attempt of removing these adverse pollutants out of the ecosystem, many have come with various methods such as wet oxidative, immobilization, hydrodechlorination and oxidation degradation but the methods stated have been reported to possess several disadvantages such as time and cost ineffective and produce large amount of by-products [4].

Advanced Oxidation Processes (AOP) is a highly potential method in removal of micropollutants where use light irradiation and catalyst to produce reactive oxygen species (ROS) such as hydroxyl radicals ( $\left.{ }^{\circ} \mathrm{OH}\right)$ [5]. It also has a ability to transform pollutants in a large amount at a time into a more durable and harmless compounds [6]. Photocatalysis is one of the AOPs which involve a chemical reaction driven by light absorption on photocatalyst without having any chemical changes during and after the reaction. This method uses various semiconductor such as $\mathrm{TiO}_{2}, \mathrm{ZnO}, \mathrm{SnO}, \mathrm{ZnS}, \mathrm{CuO}$ and $\mathrm{Fe}_{2} \mathrm{O}_{3}$ [4,7]. Among them, $\mathrm{TiO}_{2}$ has been widely used as photocatalyst in photocatalysis due to its potentials of being potent and stable even under exposure of light [8]. However, $\mathrm{TiO}_{2}$ has high electron-hole recombination rate of photodegenerated hole-electron pairs which decreased it efficiency [9]. Thus, many approaches have been studied to overcome this problem and synthesis of $\mathrm{TiO}_{2}$ in mesoporous structure for enhancing its efficiency is one of the alternatives [10].

There are various method can be used to synthesis mesoporous materials such as sol-gel, microwave-assisted, hydrothermal and sonochemical. Among them, microwave-assisted method offer a simple, clean, quick reaction, only need short time to reach the appropriate reaction temperature and its particle morphology can be controlled [11]. It also has been proven can be used as alternative to prepare oxide, hydroxide and sulfide nanoparticles [12]. Thus, in this study we intend to synthesis $\mathrm{TiO}_{2}$ as mesoporous titania nanoparticles (MTN) using microwave-assisted method and compare its photoactivity in degradation of 2-chlorophenol (2-CP) under UV light with P25 as commercial $\mathrm{TiO}_{2}$. It is expected that the microwave-assisted method is the potential method for synthesis of high surface mesoporous material and the photocatalytic performance of MTN is comparable with P25. 


\section{MATERIALS AND METHODS}

\section{Chemicals}

Titanium (IV) butoxide, Degussa P25 (commercial $\mathrm{TiO}_{2}$ ), Pluronic (F127), 2-propanol and hydrochloric acid ( $\left.\mathrm{HCl}\right)$ and sodium hydroxide were purchased from Sigma-Aldrich. Acetone, 2-chlorophenol (2-CP), 28\% ammonia solution and methanol were acquired from MERCK, Malaysia. All these chemicals were of analytical grade and were used without any further purification.

\section{Catalyst Preparation}

Mesoporous titania nanoparticles (MTN) were prepared via microwave-assisted method. F127 surfactant was dissolved in distilled water, propanol and $28 \%$ ammonia solution before stirring for 30 min at $323 \mathrm{~K}$ in water bath. After $30 \mathrm{~min}$, titanium (IV) butoxide was added into the mixture and the temperature of water bath was increased to $353 \mathrm{~K}$ before continuing stirring for another $2 \mathrm{~h}$. The white mixture solution was then transferred into beaker to continue the synthesis process in the microwave. The mixture was under microwave heating with $0.56 \mathrm{Wg}^{-1}$ of power density for $2 \mathrm{~h}$ to allow the formation of sol-gel. After $2 \mathrm{~h}$, the sol-gel was collected and dried overnight in an oven before calcined at $873 \mathrm{~K}$ for $3 \mathrm{~h}$.

\section{Characterization}

The crystalline structures of these catalysts were identified using a Bruker Advance D8 X-ray powder diffractometer (XRD) with $\mathrm{Cu} \mathrm{K \alpha}$ radiation $\left(\lambda-1.5418 \AA\right.$ ) at $2 \theta$ angle ranging from $20^{\circ}$ to $65^{\circ}$. The functional groups of the catalysts were determined using FTIR spectroscopy (Perkin Elmer Spectrum GX FTIR Spectrometer) with IR absorbance data obtained from 390 to $3800 \mathrm{~cm}^{-1}$. The band gap of the catalysts were identified using UV-Vis DRS (Perkin Elmer Lambda 900) spectrophotometer at room temperature. The surface area measurement of the catalysts were determined by Brunnauer-Emmett-Teller (BET) method. Prior to measurement, both catalysts were degassed at $300{ }^{\circ} \mathrm{C}$ and $0.1 \mathrm{~Pa}$.

\section{Photocatalytic degradation of 2-chlorophenol}

The photoactivity of the catalysts was tested on the degradation of 2-CP. The photocatalytic experiments were carried out in a batch reactor equipped with UV lamp (36 Watt) which was used as the source of light. $0.0375 \mathrm{~g}$ of catalyst was added in $200 \mathrm{~mL}$ of $70 \mathrm{ppm}$ 2-CP solution. The solution was stirred for $1 \mathrm{~h} 30 \mathrm{~min}$ in the dark to achieve adsorption desorption equilibrium followed by $6 \mathrm{~h} 30 \mathrm{~min}$ under light irradiation with continuous stirring. The initial $\mathrm{pH}$ of the solution was $\mathrm{pH} 5$ and the reaction was conducted at $303 \mathrm{~K}$. The concentration of the 2-CP in the solution prior to irradiation was used as the initial value for the 2-CP degradation measurement.

Aliquots of $2 \mathrm{~mL}$ were collected during the reaction at intervals of $30 \mathrm{~min}$ and centrifuged in a Beckman Coulter Microfuge 16 at $15000 \mathrm{rpm}$ for $10 \mathrm{~min}$ before being analysed by UV-Vis spectrophotometer (Shimadzu Corp, UV-2600 UV-Vis Spectrophotometry) for the residual concentration of 2-CP. The adsorption band of 2-CP was taken $275 \mathrm{~nm}$ and the degradation percentage was calculated using the following equation:

$$
\text { Degradation }(\%)=\frac{\mathrm{C}_{0}-\mathrm{C}_{\mathrm{t}}}{\mathrm{C}_{0}} \times 100
$$

where $\mathrm{C}_{0}$ and $\mathrm{C}_{\mathrm{t}}$ are the initial concentration of 2-CP and the concentration at time t, respectively.

\section{EXPERIMENTAL RESULTS}

Figure 1(A) shows the XRD patterns of P25 and MTN. A series of P25 and MTN at $25^{\circ}, 38^{\circ}, 39^{\circ}, 48^{\circ}, 54^{\circ}, 56^{\circ}$ and $63^{\circ}$ are consistent with peaks of $\mathrm{TiO}_{2}$ anatase [13]. However, P25 illustrates a series of XRD peaks for rutile at $28^{\circ}, 55^{\circ}$ and $57^{\circ}$. Based on the XRD patterns, both catalysts demostrated sharp peaks indicated high crystallinity in their structures. The particle size of P25 and MTN are 4.8 and $1.8 \mathrm{~nm}$, respectively, which were calculated from Debye-Scherrer equation:

$$
D=\frac{k \lambda}{\beta \cos \theta}
$$

where $\mathrm{D}$ is the particle size, $\lambda$ is the wavelength of the $\mathrm{X}$-ray radiation $(\mathrm{Cu} \mathrm{K}=0.1542 \mathrm{~nm}), k$ is the shape factor $(k=0.94)$, $\beta$ is the line width at half-maximum height, and $\theta$ is the angular position of the peak maximum, $2 \theta=25^{\circ}$.

Figure 1B shows the FTIR spectra of the catalysts. Both catalysts showed moderate bands at 3430 and $1640 \mathrm{~cm}^{-1}$ which were attributed to the $\mathrm{O}-\mathrm{H}$ stretching and $\mathrm{OH}$ vibration of the surface-adsorbed water, respectively [14]. It is belive that the presence of $\mathrm{OH}$ groups on the catalyst surface possibly will help to enhance the catalytic acivity [15]. While, the band at $450 \mathrm{~cm}^{-1}$ was assigned to Ti-O-Ti vibration modes. 

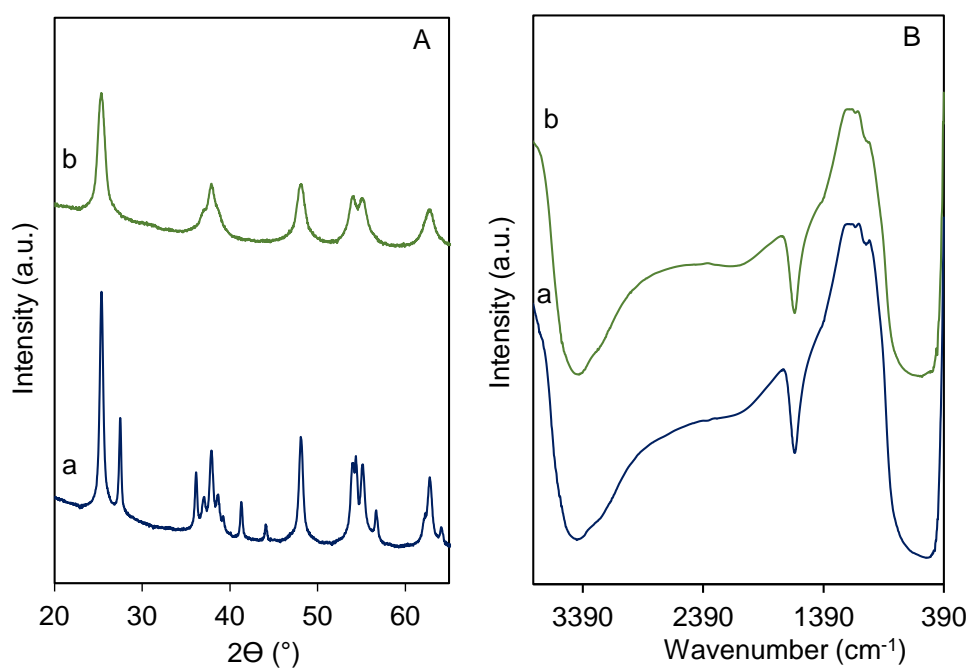

Figure 1. (A) X-ray diffraction patterns and (B) FTIR spectra of (a) P25 and (b) MTN.

The band gap of each catalyst was measured using UV-Vis DRS and Figure 2A and 2B shows the Kebulka-Munk spectra for both catalysts. The band gap for P25 and MTN are 3.0 and $3.2 \mathrm{eV}$, respectively. The band gap energy for both catalysts illustrated that both catalysts will properly work under UV light irradiation instead of visible light. In addition, the surface area for P25 and MTN were 42 and $75 \mathrm{~m}^{2} \mathrm{~g}^{-1}$, respectively. This result illustrated that the enhancement in heat distribution during the hydrolysis and condensation under microwave-assisted method help to increase the framework cross-linking for improvement in the growth of smaller pores which also explain the formation of small particle size of MTN [16]. This phenomenon was confirmed by the pore distribution (Figure 2C) for both catalysts which shows that MTN was successfully synthesized as mesoporous material when most of the formation of pores in the range 2 to $20 \mathrm{~nm}$.
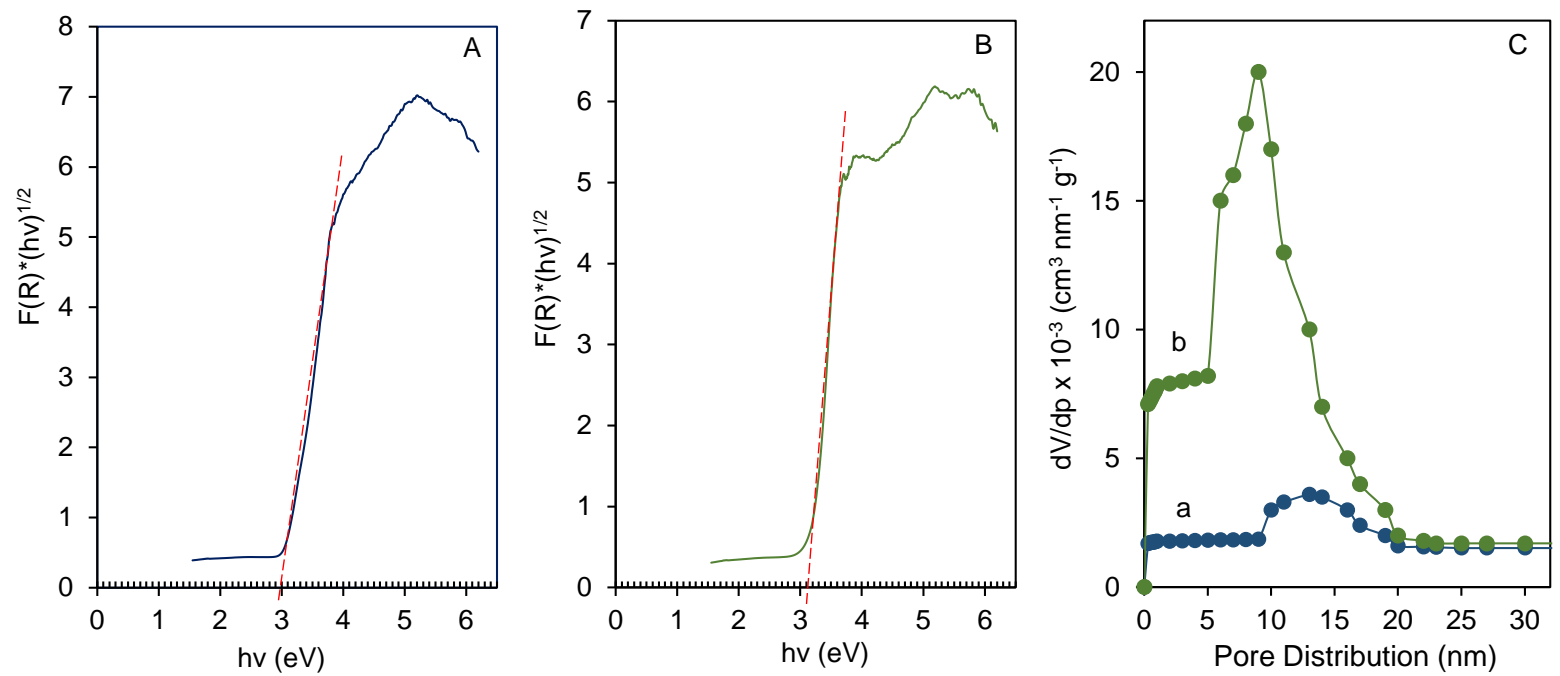

Figure 2. Kebulka-Munk spectra of (A) P25 and (B) MTN and (C) Pore distribution of (a) P25 and (b) MTN.

The catalytic performance of the catalysts was tested on degradation of 2-CP. Figure 3 shows the catalytic performance of P25 and MTN under several conditions. The photolysis of the 2-CP shows that only 4.7\% degradation was observed indicated that the 2-CP can not be degraded under UV-light without the presence of catalyst. The role of UV-light was also being investigated by undergoes the degradation of 2-CP using the catalysts in the absence of UV-light. The results demostrated that P25 and MTN only degraded 5\% and 4.8\%, respectively of 2-CP. In contrast, both catalysts successfully degraded $100 \%$ of 2-CP under UV light irradiation for $8 \mathrm{~h}$ indicating the necessity of light in this process [10]. This results demostrated that MTN showed comparable performance to P25 eventhough has slighly higher band gap value. The photocatalysis of both catalysts was tested at $\mathrm{pH} 5 . \mathrm{pH} 5$ was selected as the $\mathrm{pH}$ of solution because $\mathrm{pH}_{\mathrm{PZC}}$ for both catalysts were $6.5(\mathrm{P} 25)$ and $6.4(\mathrm{MTN})$ illustrated that strong electrostatic field that existed in between the 2-CP that carried negative charges and catalysts that carried positive charges influenced the activity of the catalysts because any $\mathrm{pH}$ lower than this value will demonstrate that the surface of the catalyst is positively charged. In addition, the high surface area of MTN probably contributed in the photocatalytic performance where provide enough surface to allow the 2-CP to adsorp on the catalyst before undergoes degradation process. 


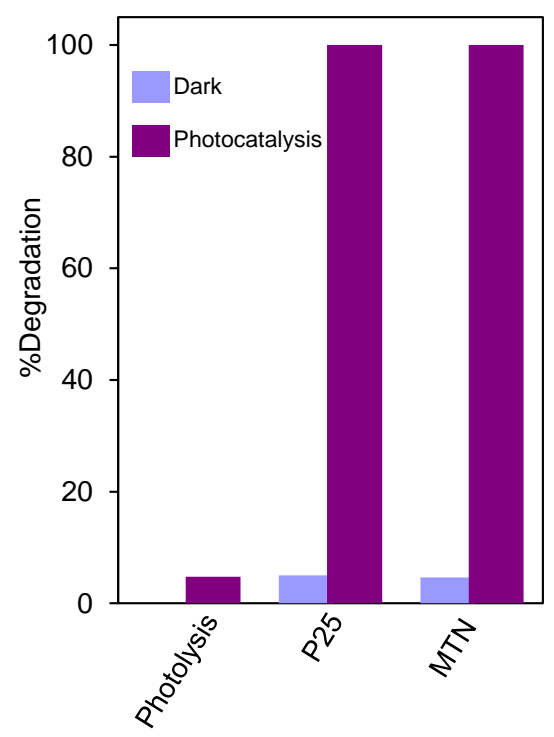

Figure 3. Catalytic performance of $\mathrm{P} 25$ and $\mathrm{MTN}\left(\mathrm{C}_{2-\mathrm{CP}}=10 \mathrm{mg} \mathrm{L}^{-1}, \mathrm{pH}=5, \mathrm{~W}=0.075 \mathrm{~g} \mathrm{~L}^{-1}, t=8 \mathrm{~h}, \mathrm{~T}=303 \mathrm{~K}\right)$.

\section{CONCLUSION}

This study reports the potential of mesoporous titania naoparticles in photocatalytic degradation of 2-chlorophenol. Mesoporous titania nanoparticles was successfully prepared by microwave-assisted menthod and the properties as well as photoactivity of MTN was compared with degussa P25 (commercial $\mathrm{TiO}_{2}$ ). Both catalysts were characterized by XRD, FTIR, UV-Vis DRS and surface area analysis. Based on BET and XRD results, MTN has higher surface area and lower particle size compare with P25. The adsorption study (without the presence of UV-light) for both catalysts illustrated that both catalysts have low degradation with only have less than 5\% degradation of 2-CP. However, $100 \%$ degradation was achieved by both catalysts when the degradation under UV-light indicated the important of light in the process. In addition, the 2-CP was successfully degraded completely under UV light irradiation despite of having a slightly higher band-gap value compared with P25 demonstrated that MTN shows a good potential as a photocatalyst.

\section{ACKNOWLEDGMENT}

The authors are grateful for the financial support by Ministry of Education Malaysia for Fundamental Research Grant (203.PKIMIA.6711607) and Universiti Sains Malaysia (USM) for Short Term Grant (304/PKIMIA/6315055).

\section{REFERENCES}

[1] C. C. Wang, J. R. Li, X. L., Lv, Y. Q. Zhang, and G. Guo, "Photocatalytic organic pollutants degradation in metal-organic frameworks," Energy \& Environmen. Sci. vol. 7 no. 9, pp. 2831-2867, 2014, doi: 10.1039/C4EE01299B.

[2] M. Á. Arellano-González, I. González, and A. C. Texier, "Mineralization of 2-chlorophenol by sequential electrochemical reductive dechlorination and biological processes," J. Hazard. Mater. vol. 314, pp. 181-187, 2016, doi: 10.1016/j.jhazmat.2016.04.048.

[3] J. Chun-Te Lin, K. Sopajaree, T. Jitjanesuwan, and M. C. Lu, "Application of visible light on copper-doped titanium dioxide catalyzing degradation of chlorophenols," Sep. Purif. Tech. vol. 191, pp. 233-243, 2018, doi: 10.1016/j.seppur.2017.09.027.

[4] N. F. Jaafar, A. M. M. Najman, A. Marfur, and N. W. C. Jusoh, "Strategies for the formation of oxygen vacancies in zinc oxide nanoparticles used for photocatalytic degradation of phenol under visible light irradiation" J. Photochem. Photobio. A: Chem. vol. 388, pp. 112202, 2020, doi: 10.1016/j.jphotochem.2019.112202.

[5] F. Formisano, A. Fiorentino, L. Rizzo, M. Carotenuto, L. Pucci, M. Giugni, and G. Lofrano, "Inactivation of Escherichia coli and Enterococci in urban wastewater by sunlight/PAA and sunlight $/ \mathrm{H}_{2} \mathrm{O}_{2}$ processes," Process Saf. Environmen., vol. 104, pp. 178-184, 2016, doi: 10.1016/j.psep.2016.09.003.

[6] N. A. Marfur, N. F. Jaafar, and F. H. H. Gani, "Effect of microwave power intensity on synthesis of mesoporous titania nanoparticles for degradation of 2, 4-dichlorophenol: Photoactivity performance and kinetic studies," In IOP Conference Series: Materials Science and Engineering, vol. 736, no. 3, pp. 032016, 2020, doi: 10.1088/1757-899X/736/3/032016.

[7] M. N. Chong, B. Jin, W.K. Chow, and C. Saint "Recent developments in photocatalytic water treatment technology: A review," Water Research, vol. 44, pp. 2997-3027, 2010, doi: 10.1016/j.watres.2010.02.039.

[8] N. F. Jaafar, N. A. Marfur, N. W. C. Jusoh, Y. Nagao, N. H. N. Kamarudin, R. Jusoh, and M. A. M. Iqbal, "Synthesis of mesoporous nanoparticles via microwave-assisted method for photocatalytic degradation of phenol derivatives" MJAS, vol. 23, no.3, pp. 462-471, 2019, doi: 10.17576/mjas-2019-2303-10.

[9] N. F. Jaafar, and A.A. Jalil, "Photocatalytic degradation of phenol derivatives over silver supported on mesoporous titania nanoparticles" MJAS, vol. 22, no. 5, pp. 807-816, 2018, doi: 10.17576/mjas-2018-2205-08.

[10] N. F. Jaafar, A. A. Jalil, S. Triwahyono, and N. Shamsuddin, "New insights into self-modification of mesoporous titania nanoparticles for enhanced photoactivity: effect of microwave power density on formation of oxygen vacancies and $\mathrm{Ti}^{3+}$ defects," RSC adv. Vol. 5, no.110, pp. 90991-91000,2015, doi: 10.1039/C5RA15120A. 
[11] N. H. N. Kamarudin, A. A. Jalil, S. Triwahyono, V. Artika, N. F. M. Salleh, A. H. Karim, and A. Johari, "Variation of the crystal growth of mesoporous silica nanoparticles and the evaluation to ibuprofen loading and release," J. Colloid Interface Sci., vol. 421, pp. 6-13, 2014, doi: 10.1016/j.jcis.2014.01.034.

[12] X. Zhang, Y. Wang, F. Hou, H. Li, Y. Yang, X. Zhang,... and Y. Wang, " Effects of Ag loading on structural and photocatalytic properties of flower-like ZnO microspheres,” App. Surf. Sci., vol. 391, pp. 476-483, 2017, doi: 10.1016/j.apsusc.2016.06.109.

[13] N. A. Marfur, N. F. Jaafar, and N. H. H. M. Habibullah, "Electrogenerated iron supported on mesoporous titania nanoparticles for the photocatalytic degradation of 2-chlorophenol," Comptes Rendus Chimie, vol. 22, no. 11-12, pp. 813-821, 2019, doi: 10.1016/j.crci.2019.08.006.

[14] A. Yamakata, J. J. M. Vequizo, and H. Matsunaga, "Distinctive behavior of photogenerated electrons and holes in anatase and rutile $\mathrm{TiO}_{2}$ powders," J. Phy. Chem. C, vol. 119, no. 43, pp. 24538-24545, 2015, doi: 10.1021/acs.jpcc.5b09236.

[15] R. Saravanan, E. Thirumal, V. K. Gupta, V. Narayanan, and A. Stephen, "The photocatalytic activity of ZnO prepared by simple thermal decomposition method at various temperatures," J. Molecul. Liq. vol. 177, pp. 394-401, 2013, doi: 10.1016/j.molliq.2012.10.018.

[16] Y. Sakatani, D. Grosso, L. Nicole, C. Boissière, G. J. de AA Soler-Illia, and C. Sanchez, "Optimised photocatalytic activity of grid-like mesoporous $\mathrm{TiO}_{2}$ films: effect of crystallinity, pore size distribution, and pore accessibility," J. Mater. Chem., vol. 16, no.1, pp. 77-82, 2006, doi: 10.1039/B512824M. 